\title{
Global sensitivity analysis of failure probability caused by fatigue crack propagation
}

\author{
Zdeněk Kala \\ Department of Structural Mechanics, Faculty of Civil Engineering, University of Technology, Brno, Czech Republic
}

E-mail:kala.z@fce.vutbr.cz

\begin{abstract}
The probability of failure of a load bearing steel member is investigated using a new type of global sensitivity analysis subordinated to contrasts. The main objective of the probability-oriented sensitivity analysis is structural reliability. The structural reliability methodology uses random variables as inputs. The subject of interest is the identification of those random variables that are most important when the limit state of a steel bridge member is reached. The limit state is defined by the occurrence of brittle fracture, which results from stress changes caused by multiple repeated loads. The propagation of a single-edge crack from initial to critical size is analysed using linear fracture mechanics. The failure probability and sensitivity indices are calculated using sampling-based methods. The sensitivity indices are estimated using double-nested-loop simulation of the Latin Hypercube Sampling method. New findings indicate that interaction effects among input variables strongly influence the probability of failure especially at the beginning of the operating period.
\end{abstract}

Keywords: sensitivity analysis, failure probability, steel, bridge, fracture.

\section{Introduction}

Sensitivity analysis (SA) is an effective method for quantifying the contribution of uncertainties of input variables to the uncertainties of the output variables of the mathematical model (Saltelli, Chan, \& Scott, 2004). Various approaches to calculating SA have been proposed, see state-of-the-art reviews (Iooss \& Lemaitre, 2015; Wei, Lu, \& Song, 2015; Borgonovo \& Plischke, 2016; Ferretti, Saltelli, \& Tarantola, 2016; Saltelli et al., 2019). An overview of SA methods based on multiple criteria decision-making is given in (e.g. Antucheviciene, Kala, Marzouk, \& Vaidogas, 2015; Kaklauskas et al., 2018).

Commonly implemented SA methods can be summarized as non-parametric techniques (e.g. Saltelli \& Marivoet, 1990), screening approaches (e.g. Xiao, Lu, \& Xu, 2016), Sobol's variance-based method (Sobol', 1993, 2001), moment-independent methods (e.g. Borgonovo, Castaings, \& Tarantola, 2012; Yun, Lu, Jiang, \& Zhang, 2018a), and many others.

Sobol sensitivity analysis (SSA) (Sobol', 1993, 2001) is one of the most comprehensive SA techniques, because it provides sophisticated measurements of all combinations of input effects on the output of non-linear and non-additive stochastic models. SSA consider the whole variation and interaction range of the inputs (Saltelli et al., 2004) and are therefore referred to as global SA (GSA). GSA are necessary to performing a valid SA when models feature nonlinearities and interactions, see for e.g. models in structural mechanics (e.g. Kala \& Valeš, 2017, 2018) or geotechnics (e.g. Chalmovský et al., 2017; Štefaňák, Kala, Miča, \& Norkus, 2018).

SSA defines sensitivity indices using the first two statistical moments (Saltelli et al., 2004). It seems very intuitive that for the estimation of the mean value or variance, the order of importance of the input variables may be different than for the estimation of the quantile or probability, which are the basic indicators of structural reliability. Global reliability sensitivity analysis algorithms based on the classification of model output (e.g. Yun, Lu, Zhan, \& Jiang, 2018b; Xiao \& Lu, 2017), which measure the effect of each model input variable on the failure probability, have been developed with this motivation. In cases where reliability is given by exceeding the quantile value, sensitivity measurements based directly on the quantiles of model outputs may be more appropriate (Maume-Deschamps \& Niang, 2018; Kala, 2019a; Kucherenko, Song, \& Wang, 2019).

Reliability-oriented GSA provides information on how one input variable on average influences the effect of another input variable on the failure of the structure. The basic indicator of reliability in structural mechanics is failure probability. In order to describe structural reliability, GSA should be oriented directly on failure probability rather than on the variance or quantile of the model output. 
This article focuses on reliability-oriented GSA (Kala, 2019b), which uses contrast (or loss) functions for sensitivity measurements (Fort, Klein, \& Rachdi, 2016). It can be noted that the contrast function is a very useful object in the Statistical Learning Theory (e.g. Massart, 2003), where it defines estimation procedures of some scalar or functional feature associated to a random output variable $Y$. The goal of the presented GSA is the sensitivity measurement of the failure probability as a basic indicator of reliability.

\section{Estimation of probability-oriented contrast indices}

In structural reliability analysis, the failure probability $P_{f}$ is a quantity of interest. Let us consider a computational model in the form $Y=f\left(X_{1}, X_{2}, \ldots, X_{M}\right)$, with $Y$ a scalar. The input variables $\left(X_{1}, X_{2}, \ldots, X_{M}\right)$ are supposed to be random variables described using identified probability distributions, which reflect the uncertain knowledge of material parameters, load actions, etc. Failure occurs if $Y<0$. The contrast function $\psi$ associated with

$$
P_{f}=P(Y \leq 0)
$$

can be written using parameter $\theta$ as

$$
\psi(\theta)=E(\psi(Y, \theta))=E\left(1_{Y \leq 0}-\theta\right)^{2} .
$$

The sensitivity measurement is based on the assumption that $\psi\left(\theta^{*}\right)$ is a contrast associated with a parameter $\theta^{*}$, where

$$
\theta^{*}=\underset{\theta}{\operatorname{Argmin}} \psi(\theta)
$$

Based on (Fort et al., 2016), the first order sensitivity index subordinated to the contrast (main effect) can be expressed as

$$
P_{i}=\frac{\min _{\theta} \psi(\theta)-E\left(\min _{\theta} E\left(\psi(Y, \theta) \mid X_{i}\right)\right)}{\min _{\theta} \psi(\theta)} .
$$

The parameter $\theta^{*}$ has the significance of failure probability and can be estimated from (1) using the Monte Carlo (MC) method. For the unconditional failure probability, the minimum value of contrast $\psi(\theta)$ can be estimated using $K$ runs of the MC method as

$$
\min _{\theta} \psi(\theta) \approx \frac{1}{K} \sum_{k=1}^{K}\left(1_{Y<0}-\theta^{*}\right)^{2} .
$$

For the conditional failure probability, the second member in the numerator (4) can be calculated similarly using double-nested-loop simulation of MC, where conditional failure probability $\theta^{*}$ is calculated for $K$ runs of $Y$ using random variables $X_{\sim i}$ (nested loop), where $X_{i}$ is a fixed (constant) run from $L$ runs of MC (outer loop). The second order contrast index $P_{i j}$ can be expressed as

$$
P_{i j}=\frac{\min _{\theta} \psi(\theta)-E\left(\min _{\theta} E\left(\psi(Y, \theta) \mid X_{i}, X_{j}\right)\right)}{\min _{\theta} \psi(\theta)}-P_{i}-P_{j}
$$

and estimated using MC analogously. The higher-order probability contrast indices can be expressed analogously. The sum of all sensitivity indices must be equal to one.

$$
\sum_{i} P_{i}+\sum_{i} \sum_{j>i} P_{i j}+\sum_{i} \sum_{j>i} \sum_{k>j} P_{i j k}+\ldots+P_{123 \ldots M}=1
$$

This contrast-based global sensitivity analysis of probability is referred to as PSA in the article. PSA is applied to the fatigue limit state of a steel member with a small initial edge crack.

\section{Fatigue crack propagation using linear fracture mechanics}

The failure type considered in this article is brittle fracture of the steel member. The propagation of an initial crack of magnitude $a_{0}$ to the critical crack $a_{c r}$ is analysed using linear elastic fracture mechanics. The Paris-Erdogan law (Paris \& Erdogan, 1963) relates the stress intensity factor range to sub-critical crack growth under the fatigue stress regime. 


$$
\frac{d a}{d N}=C \cdot(\Delta K)^{m}
$$

where $a$ is the crack length, $N$ is the number of loading cycles and $\mathrm{d} a / \mathrm{d} N$ is the crack propagation rate, which denotes the crack growth for a load cycle, $m$ and $C$ are material-related parameters dependent on environment, stress ratio, etc. Parameter $C$ can be calculated as

$$
\log (C)=c_{1}+m \cdot c_{2}
$$

where $c_{1}, c_{2}$ can be considered for steel grade S235 as $c_{1}=-11.141, c_{2}=-0.507$ (e.g. Kala, 2018). The range of stress intensity factor $\Delta K$ can be determined by Broek:

$$
\Delta K=\Delta \sigma_{E} \cdot \sqrt{\pi a} \cdot F(a)
$$

where $\Delta \sigma_{E}$ is the equivalent quasi-constant stress range. The calibration function $F(a / W)$ for pure bending is used. $F(a / W)$ from experimental research (Seitl, Miarka, \& Kala, 2018) is in the form:

$$
F\left(\frac{a}{W}\right)=1.114\left[1-0.806\left(\frac{a}{W}\right)+2.4704\left(\frac{a}{W}\right)^{2}+1.01643\left(\frac{a}{W}\right)^{3}\right], \frac{a}{W} \in[0.01,0.5] .
$$

The resistance $R_{c r}$ can be calculated as the accumulation of damage related to the growth from $a_{0}$ into $a_{c r}$.

$$
R_{c r}=\int_{a_{0}}^{a_{c r}} \frac{d a}{[f(a) \cdot \sqrt{\pi \cdot a}]^{m}}
$$

Failure occurs when $R_{c r}$ is equal to the load effects $A_{t}$.

$$
A_{t}=\Delta \sigma_{E}^{m} \cdot C \cdot N
$$

where $N$ is the total number of cycles reached at the time the crack reaches its critical length $a_{c r}=0.5 \cdot W$. The value of action $A_{t}$ is a function of time, because the cumulative effect of traffic $N$ is a non-decreasing function in time. The reliability function can then be written as:

$$
Y=R_{c r}-A_{t},
$$

where $W$ is the specimen width in the direction of crack propagation.

\section{Computation of sensitivity indices}

The initial length $a_{0}$ of the single-edge crack is considered using a log-normal probability density function (pdf) with mean value of $0.2 \mathrm{~mm}$ and standard deviation of $0.06 \mathrm{~mm}$ (Maljaars \& Vrouwenvelder, 2014; Kala, 2018), see Table 1. The other input random variables with Gauss pdf listed in Table 1 are considered acc. to (Kala, 2017, 2018).

Table 1. Input random quantities

\begin{tabular}{|l|c|c|c|c|c|}
\hline \multicolumn{1}{|c|}{ Description } & Symbol & Pdf & Unit & Mean value & Coef. of variation \\
\hline Initial crack length & $a_{0}$ & log-normal & $\mathrm{mm}$ & 0.2 & 0.3 \\
\hline Specimen width & $W$ & Gauss & $\mathrm{mm}$ & 400 & 0.05 \\
\hline Paris exponent & $m$ & Gauss & - & 3 & 0.01 \\
\hline Equivalent stress range & $\Delta \sigma_{E}$ & Gauss & $\mathrm{MPa}$ & 30 & 0.1 \\
\hline Stress peaks per year & $N$ & Gauss & - & $10^{6}$ & 0.1 \\
\hline
\end{tabular}

For example, for 50 years of bridge operation random variable $N$ has a mean value of $50 \times 10^{6}$ and variation coefficient of $\sqrt{50} \times 0.1$. The Latin Hypercube Sampling (LHS) method (McKey, Beckman, \& Conover, 1979; Iman \& Conover, 1980) is used to evaluate all sensitivity indices in the decomposition (7). Each index is estimated using $K=70 \mathrm{E} 6, L=2 \mathrm{E} 4$ of LHS runs.

The results of the sensitivity analysis are shown in Figure 1. Equivalent stress range $\Delta \sigma$ has a crucial influence on $P_{f}$ at both times of bridge operation, but is also involved in interactions with variables $a_{0}, m, N$, see Figure 1 . The second dominant variable is the initial crack length $a_{0}$. The sum of first and second order sensitivity indices $P_{\Delta \sigma}+P_{a 0}+P_{\Delta \sigma, a 0}$ is approximately $56 \%$ at the time of 50 years and $77 \%$ at the time of 100 years. All interaction effects 
calculated as $1-\sum_{i} P_{i}$ are approximately $68 \%$ at the time of 50 years and $30 \%$ at the time of 100 years. Specimen width $W$ is identified as a non-influential variable, which may have any variation coefficient not influencing $P_{f}$, practically zero variation coefficient can be considered. The critical length $a_{c r}=0.5 \cdot W$ should also have a negligible influence on $P_{f}$ (similarly as $W$ ). However, this should be verified for other statistical models of $a_{c r}$, whose variation coefficients can be discussed with higher values or other types of pdfs.
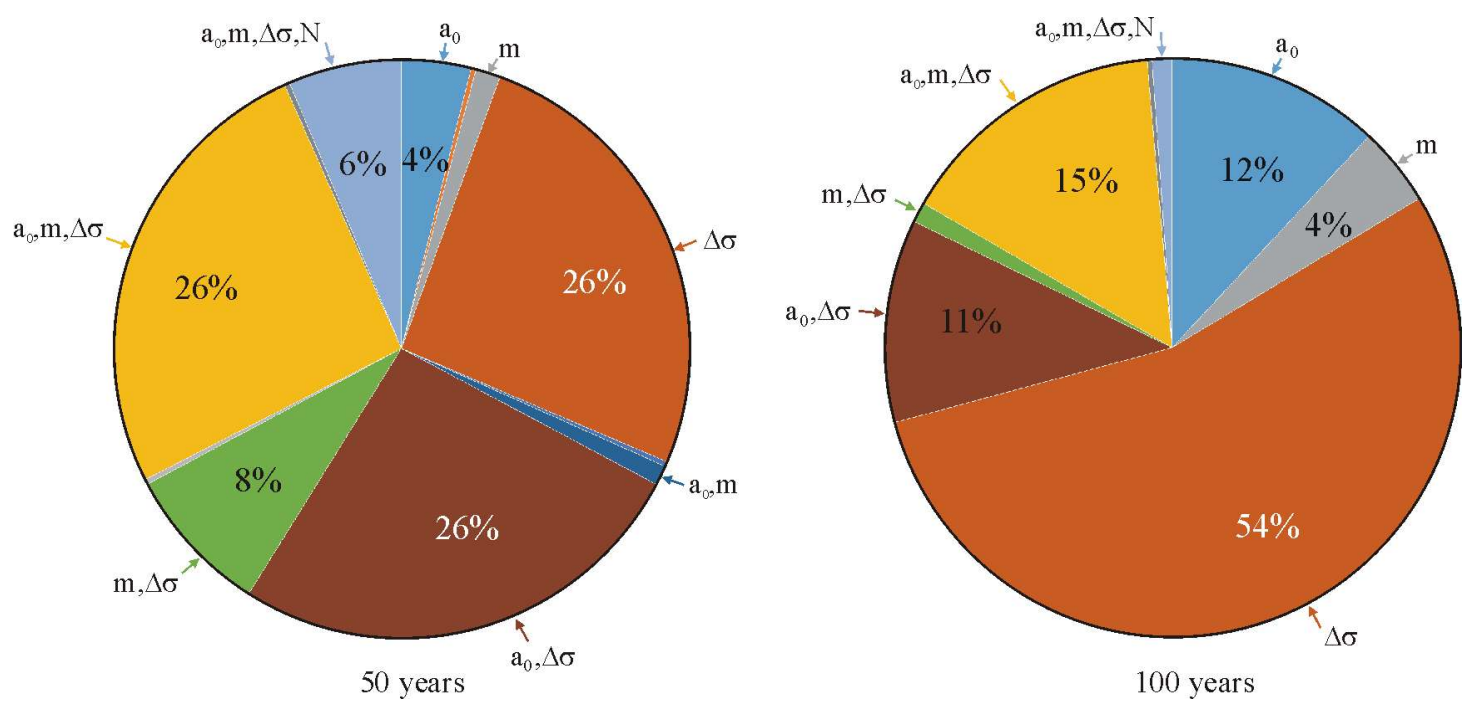

Figure 1. Global sensitivity indices

PSA has identified relatively strong interaction effects, which are unusual in SSA of limit states of single members (e.g. Kala \& Valeš, 2017, 2018). On the contrary, strong interaction effects can easily be detected by SSA in frame structures (e.g. Kala, 2012). For the probability assessment of the limit states, it may be interesting to compare the results of SSA and PSA in cases where SSA identifies non-negligible interactions effects.

Currently, there are many types of GSA and it is not easy to find time-proven practices suitable for the given type of task (Saltelli et al., 2019). Reliability-oriented GSA must be based on a sensitivity measurement that directly analyses the probability of failure as a basic indicator of reliability. It may be noted that other suitable methods exist for this purpose (e.g. Xiao \& Lu, 2017; Wang, Xiao, \& Lu, 2019) and probability analysis is not the only method for measuring structural reliability. Structural design according to the EUROCODE standard is based on the so-called semi-probability method of partial safety factors, in which the design values can be verified using design quantiles. Coherent reliability-oriented GSA should ensure that probability-oriented and quantile-oriented sensitivity indices form pairs based on the same theoretical basis. Sensitivity indices subordinated to the contrast (Fort et al., 2016) are proving to be one of the promising ways of finding coherent reliability-oriented GSA. However, this domain deserves much more additional work in order to make reliability-oriented GSA a useful and practical tool.

\section{The equivalent stress range - some observations}

The equivalent stress range $\Delta \sigma_{E}$ is identified as the dominant variable having the most effect on $P_{f}$, see Figure 1. In general, if the histogram of variable stress range amplitude $\Delta \sigma$ is known, the equivalent stress range $\Delta \sigma_{E}$ can be calculated as:

$$
\Delta \sigma_{E}=\left(\frac{1}{H} \sum_{h=1}^{H} \Delta \sigma_{h}^{m}\right)^{\frac{1}{m}},
$$

where $\Delta \sigma_{h}$ are random realizations of the variable stress range (e.g. Kala, 2008), which are obtained from measurements of a real bridge. Theoretically, exponential or Rayleigh pdfs can be assumed for $\Delta \sigma$. Rayleigh pdf is a one-parameter distribution (see Figure 2), which can be written as:

$$
f(\Delta \sigma ; p)=\frac{\Delta \sigma}{p^{2}} e^{-\frac{1}{2}\left(\frac{\Delta \sigma}{p}\right)^{2}} .
$$


Let $\Delta \sigma_{E}$ have a Gauss pdf according to Table $1, \Delta \sigma$ has a Rayleigh pdf with parameter $p$ and $m=3$. For each $\Delta \sigma$, parameter $p$ can be found by inverse analysis from (15) using the bisection method. The cut-off limit below which stress ranges of the design spectrum do not contributc to the calculated cumulative damage is not applied in this study. For 50 thousand LHS runs of $\Delta \sigma_{E}$ we can find 50 thousand random realizations of $p$, see blue line on Figure 3. The dependence between $\Delta \sigma_{E}$ and $p$ is linear. Therefore, if $\Delta \sigma_{E}$ has a Gauss pdf then $p$ also has a Gauss pdf, see Figure 3. The statistical uncertainty of $\Delta \sigma_{E}$ can thus be transformed to the uncertainty of parameter $p$ of the Rayleigh pdf, see Figure 3.

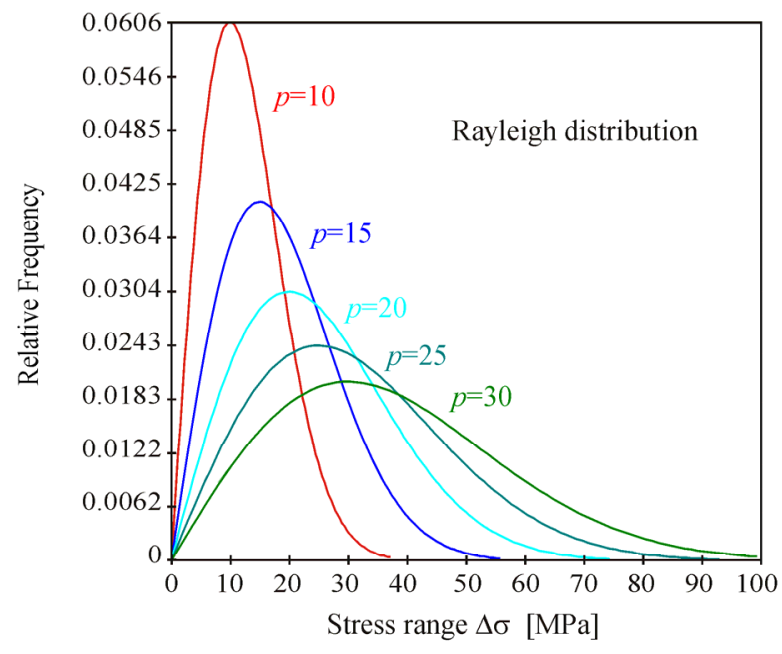

Figure 2. Examples of Rayleigh pdf realizations

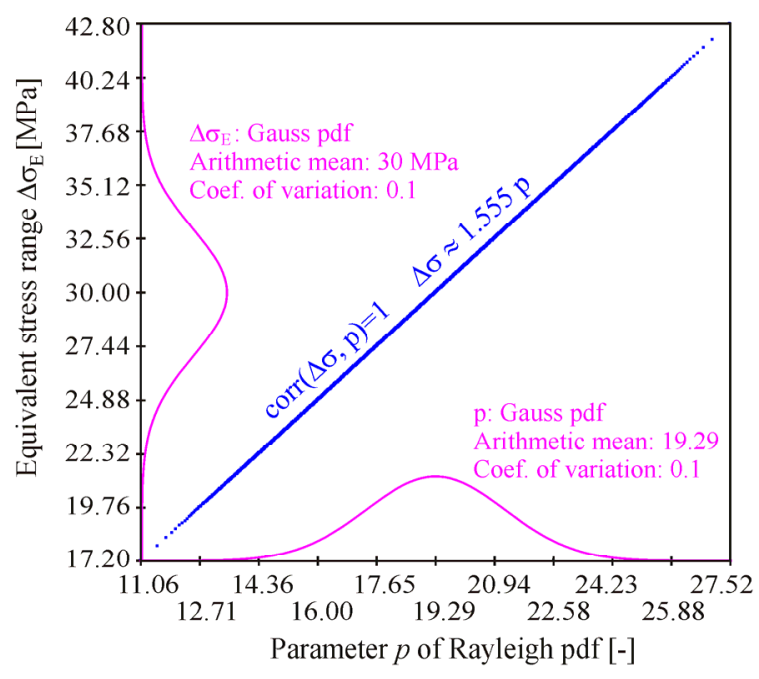

Figure 3. The relationship between $p$ and $\Delta \sigma_{\mathrm{E}}$

Equivalent stress range and average daily truck traffic can be computed based on the created stress-range histogram from long-term monitoring program (Kwon \& Frangopol, 2010). Due to loading uncertainties, a probabilistic approach considering random uncertainty for load effects can be used to predict stress ranges during fatigue lifetime (Kwon \& Frangopol, 2010). However, lack of knowledge about the type of pdf of $\Delta \sigma$ shifts the aleatoric uncertainty of $\Delta \sigma_{E}$ to the category of epistemic uncertainties. Then it can be discussed whether the uncertainty of $\Delta \sigma_{E}$ is aleatoric or epistemic. Epistemic uncertainty is also known as systematic uncertainty, and is due to things one could in principle know but doesn't in practice.

In the presented example, finding epistemic uncertainty is significant, because it is pertinent to the input random variable $\Delta \sigma_{E}$, which was identified as crucial by sensitivity analysis. Reducing epistemic uncertainties to aleatoric uncertainties can be based both on the collection of information on the numbers and weight of the axles of moving vehicles as well as on model-based prediction of the intensity of traffic flow and static analyses of $\Delta \sigma$ processed into histograms.

\section{Conclusions}

The example presented in this article shows that sensitivity indices subordinated to contrasts effectively analyse the influence of input variables on the probability of failure. Such global sensitivity analysis can be useful for problems in which the analysts are interested in the ranking of inputs contributing to the extreme values of the failure probability. Such probability-oriented sensitivity measurements are much more valuable for structural reliability than other types of sensitivity analyses, which monitor the influence of input variables on the variability of the model output.

The sensitivity indices subordinated to the contrast contain both individual sensitivity index and interaction sensitivity index. These contrast-based global sensitivity analyses of failure probability show interaction effects that are unusually strong in comparison with some results of Sobol's sensitivity analysis aimed at the reliability of structures. The failure probability is most influenced by the main and interaction effects of the equivalent stress range and the initial crack length in both analysed times. The evaluation of the sensitivity analysis at the time of 50 and 100 years has shown that the sizes of the sensitivity indices and the ranking of importance of the input variables change over the lifetime of the bridge. At the time of 100 years, the interaction effects between input variables are weaker than at the time of 50 years.

It can be noted that the evaluation of global sensitivity analysis of small probabilities of failure, which occur after a short period of operation of a bridge, has high demands on computer CPU time. With this motivation, it would be useful to make comparisons with other sensitivity analysis methods focusing, for example, on the reliability index, which is a certain alternative to the probability of failure, but has lower demands on the computer CPU time. 


\section{Acknowledgements}

This work was supported by the The Czech Science Foundation (GACR) [grant numbers 17-01589S].

\section{References}

Antucheviciene, J., Kala, Z., Marzouk, M., \& Vaidogas, E. R. (2015). Solving civil engineering problems by means of fuzzy and stochastic MCDM methods: Current state and future research. Mathematical Problems in Engineering, 2015, 1-16. https://doi.org/10.1155/2015/362579

Borgonovo, E., \& Plischke, E. (2016). Sensitivity analysis: A review of recent advances. European Journal of Operational Research, 248(3), 869-887. https://doi.org/10.1016/j.ejor.2015.06.032

Borgonovo, E., Castaings, W., \& Tarantola, S. (2012). Model emulation and moment-independent sensitivity analysis: An application to environmental modelling. Environmental Modelling \& Software, 34, 105-115. https://doi.org/10.1016/j.envsoft.2011.06.006

Chalmovský, J., Štefaňák, J., Miča, L., Kala, Z., Skuodis, Š., Norkus, A., \& Žilionienė, D. (2017). Statistical-numerical analysis for pullout tests of ground anchors. Baltic Journal of Road and Bridge Engineering, 12(3), 145-153. https://doi.org/10.3846/bjrbe.2017.17

Ferretti, F., Saltelli, A., \& Tarantola, S. (2016). Trends in sensitivity analysis practice in the last decade. Science of the Total Environment, 568, 666-670. https://doi.org/10.1016/j.scitotenv.2016.02.133

Fort, J. C., Klein, T., \& Rachdi, N. (2016). New sensitivity analysis subordinated to a contrast. Communications in Statistics Theory and Methods, 45(15), 4349-4364. https://doi.org/10.1080/03610926.2014.901369

Iman, R. L., \& Conover, W. J. (1980). Small sample sensitivity analysis techniques for computer models. With an application to risk assessment. Communications in Statistics - Theory and Methods, 9(17), 1749-1842. https://doi.org/10.1080/03610928008827996

Iooss, B., \& Lemaitre, P. (2015). Uncertainty management in simulation-optimization of complex systems. New York: Springer.

Kaklauskas, A., Zavadskas, E. K., Radzeviciene, A., Ubarte, I., Podviezko, A., Podvezko, V., Kuzminske, A., Banaitis, A., Binkyte, A., \& Bucinskas, V. (2018). Quality of city life multiple criteria analysis. Cities, 72(Part A), 82-93. https://doi.org/10.1016/j.cities.2017.08.002

Kala, Z. (2008). Fuzzy probability analysis of the fatigue resistance of steel structural members under bending, Journal of Civil Engineering and Management, 14(1), 67-72. https://doi.org/10.3846/1392-3730.2008.14.67-72

Kala, Z. (2012). Geometrically non-linear finite element reliability analysis of steel plane frames with initial imperfections. Journal of Civil Engineering and Management, 18(1), 81-90. https://doi.org/10.3846/13923730.2012.655306

Kala, Z. (2017). Stochastic inverse analysis of fatigue cracks based on linear fracture mechanics. International Journal of Mathematical and Computational Methods, 2, 60-65.

Kala, Z. (2018). Probabilistic modelling of fatigue crack - Some observations about conditional probability. International Journal of Mechanics, 12, 121-130.

Kala, Z. (2019a). Quantile-oriented global sensitivity analysis of design resistance. Journal of Civil Engineering and Management, 25(4), 297-305. https://doi.org/10.3846/jcem.2019.9627

Kala, Z. (2019b). Global sensitivity analysis of reliability of structural bridge system. Engineering Structures, 194, 36-45. https://doi.org/10.1016/j.engstruct.2019.05.045

Kala, Z., \& Valeš, J. (2017). Global sensitivity analysis of lateral-torsional buckling resistance based on finite element simulations. Engineering Structures, 134, 37-47. https://doi.org/10.1016/j.engstruct.2016.12.032

Kala, Z., \& Valeš, J. (2018). Imperfection sensitivity analysis of steel columns at ultimate limit state. Archives of Civil and Mechanical Engineering, 18(4), 1207-1218. https://doi.org/10.1016/j.acme.2018.01.009

Kucherenko, S., Song, S., \& Wang, L. (2019). Quantile based global sensitivity measures. Reliability Engineering and System Safety, 185, 35-48. https://doi.org/10.1016/j.ress.2018.12.001

Kwon, K., \& Frangopol, D. M. (2010). Bridge fatigue reliability assessment using probability density functions of equivalent stress range based on field monitoring data. International Journal of Fatigue, 32(8), 1221-1232. https://doi.org/10.1016/j.ijfatigue.2010.01.002

Maljaars, J., \& Vrouwenvelder, T. (2014). Fatigue failure analysis of stay cables with initial defects: Ewijk bridge case study. Structural Safety, 51, 47-56. https://doi.org/10.1016/j.strusafe.2014.05.007

Massart, P. (2003). Concentration inequalities and model selection. New York: Springer.

Maume-Deschamps, V., \& Niang, I. (2018). Estimation of quantile oriented sensitivity indices. Statistics and Probability Letters, 134, 122-127. https://doi.org/10.1016/j.spl.2017.10.019

McKey, M. D., Beckman, R. J., \& Conover, W. J. (1979). A comparison of the three methods of selecting values of input variables in the analysis of output from a computer code. Technometrics, 21(2), 239-245. https://doi.org/10.1080/00401706.1979.10489755

Paris, P. C., \& Erdogan, F. (1963). A critical analysis of crack propagation laws. Journal of Basic Engineering, 85(4), 528-534. https://doi.org/10.1115/1.3656900

Saltelli, A., \& Marivoet, J. (1990). Non-parametric statistics in sensitivity analysis for model output: A comparison of selected techniques. Reliability Engineering \& System Safety, 28(2), 229-253. https://doi.org/10.1016/0951-8320(90)90065-U 
Saltelli, A., Aleksankina, K., Becker, W., Fennell, P., Ferretti, F., Holst, N., Li, S., \& Wu, Q. (2019). Why so many published sensitivity analyses are false: A systematic review of sensitivity analysis practices. Environmental Modelling \& Software, 114, 29-39. https://doi.org/10.1016/j.envsoft.2019.01.012

Saltelli, A., Chan, K., \& Scott, E. M. (2004). Sensitivity analysis ( $2^{\text {nd }}$ ed). Chichester: John Wiley \& Sons Ltd.

Seitl, S., Miarka, P., \& Kala, Z. (2018). Geometry functions for edge cracks in steel bridge under three- and four- point bending with various span. Transactions of the VSB - Technical University of Ostrava, Civil Engineering Series, 18(2), 1-6. https://doi.org/10.31490/tces-2018-0015

Sobol', I. M. (1993). Sensitivity analysis for non-linear mathematical models. Mathematical Modelling and Computational Experiment, 1, 407-414.

Sobol', I. M. (2001). Global sensitivity indices for nonlinear mathematical models and their Monte Carlo estimates. Mathematics and Computers in Simulation, 55(1-3), 271-280. https://doi.org/10.1016/S0378-4754(00)00270-6

Štefaňák, J., Kala, Z., Miča, L., \& Norkus, A. (2018). Global sensitivity analysis for transformation of Hoek-Brown failure criterion for rock mass. Journal of Civil Engineering and Management, 24(5), 390-398. https://doi.org/10.3846/jcem.2018.5194

Wang, Y., Xiao, S., \& Lu, Z. (2019). An efficient method based on Bayes' theorem to estimate the failure-probability-based sensitivity measure. Mechanical Systems and Signal Processing, 115, 607-620. https://doi.org/10.1016/j.ymssp.2018.06.017

Wei, P., Lu, Z., \& Song, J. (2015). Variable importance analysis: A comprehensive review. Reliability Engineering \& System Safety, 142, 399-432. https://doi.org/10.1016/j.ress.2015.05.018

Xiao, S., \& Lu, Z. (2017). Structural reliability sensitivity analysis based on classification of model output. Aerospace Science and Technology, 71, 52-61. https://doi.org/10.1016/j.ast.2017.09.009

Xiao, S., Lu, Z., \& Xu, L. (2016). A new effective screening design for structural sensitivity analysis of failure probability with the epistemic uncertainty. Reliability Engineering \& System Safety, 156, 1-14. https://doi.org/10.1016/j.ress.2016.07.014

Yun, W., Lu, Z., Jiang, X., \& Zhang, L. (2018a). Borgonovo moment independent global sensitivity analysis by Gaussian radial basis function meta-model. Applied Mathematical Modelling, 54, 378-392. https://doi.org/10.1016/j.apm.2017.09.048

Yun, W., Lu, Z., Zhang, L., \& Jiang, X. (2018b). An efficient global reliability sensitivity analysis algorithm based on classification of model output and subset simulation. Structural Safety, 74, 49-57. https://doi.org/10.1016/j.strusafe.2018.04.003 